Objective To determine changes in incidence of CP and motor disorders in preterm children since 2004, and to identify associated factors.

Methods We included all infants born $<32$ weeks, admitted to our tertiary NICU, born in 2004-2005 (period-I) and in 2008-2009 (period-II). We excluded children transferred from other tertiary NICUs, with major malformations, and neuromuscular disorders. We compared the incidence of CP and other motor disorders at the age of 2 years. To identify potential risk factors, we used the Nursery Neurobiologic Risk Score (NBRS), including $\mathrm{pH}$, ventilation, infection, convulsions, intraventricular hemorrhage, periventricular leukomalacia, and hypoglycemia.

Results 558 children were included; 269 period-I and 289 period-II. The incidence in CP was not significantly different: $5.6 \%$ vs $6.6 \%$, respectively. The number of children with other motor disorders was significantly higher in period-I than in period-II: 15 vs $5(5.6 \%$ vs $1.7 \%, p<0.05$ ). NBRS-scores were median 2.5 vs 2.0 ( $p=0.12$ ). In period-I, $\mathrm{pH}$ and infection contributed more to a higher NBRS, whereas mild periventricular leukomalacia did in period-II (all $p<0.05)$. Total and subscores of the NBRS were strongly related to CP $(p<0.01)$, apart from hypoglycemia in both periods, and ventilation and suspected infections in period-I.

Conclusions Since 2004, the incidence of CP in preterm children did not change, but rates of other motor disorders decreased, without considerable changes in associated risk factors.

\section{NEURODEVELOPMENTAL OUTCOME ONE YEAR AFTER EARLY VERSUS LATE SELECTIVE SURFACTANT TREATMENT}

doi:10.1136/archdischild-2012-302724.1239

${ }^{12}$ Eras, ${ }^{2} \mathrm{HT}$ Aksoy, ${ }^{2} \mathrm{R}$ Ozdemir, ${ }^{2} \mathrm{~N}$ Uras, ${ }^{3} \mathrm{U}$ Dilmen. ${ }^{1}$ Developmental Behavioral Pediatrics Unit; ${ }^{2}$ Neonatology, Zekai Tahir Burak Maternity and Teaching Hospital; ${ }^{3}$ Neonatology, Zekai Tahir Burak Maternity and Teaching Hospital, Yıldırım Beyazıt University Department of Pediatrics, Ankara, Turkey

Aim To investigate whether neurodevelopmental outcome at age one year might be different after early versus late rescue surfactant treatment in preterm infants.

Methods In 54 preterm infants, having gestational age between 25-30 weeks who were enrolled in a controlled trial of early versus late selective surfactant treatment (45 vs. 70 min respectively), a standardized follow up of medical history, neurodevelopmental outcome using the Bayley Scales of Infant and Toddler Development, Second Edition at 9-12 months corrected age.scales were carried out.

Results Median Mental developmental index (MDI) score was 107 for early group and 111 for late group. Median Psychomotor developmental index (PDI) score was 82 for early group and 93 for late group. Although median MDI and PDI scores were slightly higher in late poractant treatment group and neurodevelopmental impairment was higher in early rescue group than the late rescue group, this was not statistically significant.

Conclusion Our results demostrated that both early and late poractant treatment had similiar effects on the neurodevelopmental outcomes of preterm infants with RDS. In terms of neurodevelopmental outcomes there is no obvious advantage of an immediate surfactant administration in preterm infants according to our results.

\section{MOTHERS' AND HEALTH PROFESSIONALS' PERSPECTIVES OF BARRIERS AND FACILITATORS TO ATTENDANCE AT CANADIAN NEONATAL FOLLOW-UP PROGRAMS}

doi:10.1136/archdischild-2012-302724.1240

${ }^{1} \mathrm{M}$ Ballantyne, ${ }^{2 \mathrm{~K}}$ Benzies, ${ }^{3} \mathrm{~A}$ Lodha, ${ }^{4} \mathrm{P}$ Rosenbaum. ${ }^{1}$ Nursing, Faculty of Health Sciences, McMaster University, Hamilton, ON; ${ }^{2}$ Faculty of Nursing: ${ }^{3}$ Department of
Paediatrics, University of Calgary, Calgary, AB; ${ }^{4}$ Department of Paediatrics, McMaster University, Hamilton, ON, Canada

Background and Aims Neonatal Follow-Up (NFU) programs are increasingly challenged to support attendance, address family needs, and provide essential services. Up to $30 \%$ of families do not attend these programs. The aim of this research was to investigate barriers and facilitators of NFU attendance from the perspectives of health care professionals (HCPs) and mothers.

Methods A qualitative research approach using purposive maximum variation sampling was conducted. HCPs participated in focus groups; mothers in individual interviews. Descriptive analysis was conducted to determine themes. Data from HCPs and mothers were analyzed separately and then compared.

Results Participants were $20 \mathrm{HCPs}$ from 9 NFU programs and 6 mothers from $2 \mathrm{NFU}$ programs located in the most populous regions of central and western Canada. Both HCPs and mothers identified limited family resources and restrictive clinic operations as barriers. HCPs identified fear of bad news as a barrier; mothers viewed bad news as a facilitator; the need to address the issue and move forward. Both HCPs and mothers viewed vulnerability as a barrier; however, the meaning differed. HCPs reported creating vulnerability for the family by monitoring their child's development over time; whereas, mothers reported the need to protect their vulnerable child from risks (i.e., infection, weather). For mothers, the vulnerable child in combination with limited family resources was a key barrier to attendance.

Conclusions A better understanding of these perspectives may facilitate modifications to NFU programs to increase attendance, and ultimately improve outcomes for children at high risk for developmental delays and their families.

\section{ADAPTATION PROBLEMS IN VERY LOW BIRTH WEIGHT CHILDREN AT 10 YEARS OF AGE}

doi:10.1136/archdischild-2012-302724.1241

${ }^{1} \mathrm{~T}$ Fjørtoft, ${ }^{2,3} \mathrm{KH}$ Grunewaldt, 'M Sognnæs, ${ }^{2,4} \mathrm{GCC}$ Løhaugen, ${ }^{2,3,4} \mathrm{~J}$ Skranes, ${ }^{5,6} \mathrm{KAl}$ Evensen. 'Clinical Services, St. Olavs University Hospital; ${ }^{2}$ Laboratory Medicine, Children's and Women's Health, Norwegian University of Science and Technology; ${ }^{3}$ Paediatrics, St. Olav University Hospital, Trondheim; 'Paediatrics, Sørlandet Hospital, Arendal; ${ }^{5}$ Public Health and General Practice, Norwegian University of Science and Technology; ${ }^{6}$ Physiotherapy, Municipality of Trondheim, Trondheim, Norway

Background Adaptive behaviour is the behaviour necessary for an individual to function safely and appropriately in daily life, both at a personal and social level. The Vineland Adaptive Behavior Scales (VABS) has been used to describe an individual's adaptive behaviour as reported by caregivers.

Objective To compare skills of communication, daily living and socialisation as well as the total behaviour score in very low birth weight (VLBW) children with a control group at ten years of age.

Design/methods In this follow-up study, 39 children with birth weight below 1500 grams, including 10 children with cerebral palsy (CP), and 31 term born control children were evaluated by VABS at ten years of age. The informants were parents, mostly mothers. In VABS, adaptive behaviour is expressed as total adaptive behavior composite score based on the three subscales: Communication, daily living skills and socialisation.

Results The mean total adaptive behaviour composite score in the VLBW group was 85.7 (SD 16.8) compared with 105.5 (SD 17.5) in the control group $(p<0.001)$. All three subscales; communication, daily living skills and socialisation were significantly lower in the VLBW group than in the control group. Excluding children with CP, the total adaptive behavior composite score was 90.6 (SD 14.5) in the VLBW, still significantly lower than in the control group $(\mathrm{p}=0.001)$. 
Conclusions The findings of this study indicate that VLBW children as a group have more adaptation challenges than their peers born at term. This was still the case when children with CP were excluded.

\section{EVALUATION OF FEEDING-EDUCATION PROGRAMME FOR CHILDREN WITH CEREBRAL PALSY AT INPATIENT PEDIATRIC UNIT: FROM CAREGIVERS' PERCEPTION}

doi:10.1136/archdischild-2012-302724.1242

R Hoque. Dept. of Occupational Therapy, Centre for the Rehabilitation of the Paralyzed, Dhaka, Bangladesh

Objective Evaluate the feeding-education programme of the Paediatric Inpatient Unit of CRP, Savar, Dhaka, from perceptions of caregivers of children with cerebral palsy, who are attending the feeding-education programme.

Methodology The study was conducted using phenomenological method in qualitative approach. Caregivers of children with cerebral palsy were the study participants who stay with their children in the Paediatric Inpatient Unit of CRP and attend the feeding-education programme. Nineteen participants were selected using purposive sample. Data were generated through 7 individual interviews and 3 focus-group discussions and observations within 2 months. Each focus group consisted of 4 participants. Face-to-face interviews were conducted using a semi-structured questionnaire. Data were analyzed using content analysis under category, code and preparing theme for result.

Results The results indicate that the caregivers of children with cerebral palsy felt that the feeding education programme was very important for them and their children. They mentioned that this programme improved the feeding performance of their children and enhanced their learning.

Conclusion The results suggest that the feeding education programme has the potential to make a valuable contribution to education. Caregivers were very satisfied with occupational therapists for their way of teaching, demonstration, and repetition of information for better understanding, which directly help caregivers manage their children's feeding and reduce their stress.

\section{ETIOLOGY AND SEVERITY OF HL, SCHOOL TYPE AND MODE OF COMMUNICATION IN NICU GRADUATES AT 3-5 YEARS OF AGE}

doi:10.1136/archdischild-2012-302724.1243

'EA van de Ven, 'HLM van Straaten, ${ }^{2} \mathrm{AM}$ Oudesluys-Murphy, ${ }^{2} \mathrm{AMH}$ Korver. ${ }^{1} \mathrm{Dept}$ Neonatology, Isala Clinics, Zwolle; 2 Pediatrics, Leiden University Medical Center, Leiden The Netherlands

Background Little is known about follow up of NICU graduates with permanent hearing loss (HL) following introduction of neonatal hearing screening.

Aim Evaluation of etiology, severity of HL, school type and mode of communication in NICU graduates.

Methods All NICU graduates with HL identified at the age of 3-5 years at the speech and hearing centres in the Netherlands were included. Results of 2 stage AABR neonatal hearing screening, medi$\mathrm{cal}$ and audiologic follow up as well as performance measures for school type and primary mode of communication were evaluated.

Results Included were 91 newborns [BW 1990 g (sd 1070); GA 32.9 wks (sd 5.3)]. Of those $5.6 \%$ passed neonatal hearing screening. In $44.3 \%$ moderate HL (40-60 dB), in $23.9 \%$ severe $(60-80 \mathrm{~dB})$ and in $31.8 \%$ profound $\mathrm{HL}(>80 \mathrm{~dB}$ ) was established.

In 33/91 cases parental consent was obtained for adjuvant follow up data. In $30.3 \% \mathrm{HL}$ was of hereditary origin, in $24.2 \% \mathrm{HL}$ was caused by asphyxia, in $9.1 \%$ by CMV-infection. In 8 cases $(24.2 \%)$ no cause was identified.
Up to $71.4 \%$ of NICU graduates attended schools for deaf and hard of hearing, $3.6 \%$ different special education, while $25 \%$ visit regular education. In $82.8 \%$ a combination of spoken en signed language was used for communication.

Conclusions After introduction of AABR universal neonatal hearing screening and early intervention in NICU graduates most affected children have moderate to severe HL, attend schools for deaf and hard of hearing, and use a combination of signed and spoken language at age $3-5$ years.

\section{IMPACT OF ORAL VERSUS INTRAVENOUS IBUPROFEN ON NEURODEVELOPMENTAL OUTCOME: A RANDOMISED CONTROLLED PARALEL STUDY}

doi:10.1136/archdischild-2012-302724.1244

'Z Eras, ${ }^{2} \mathrm{~T}$ Gokmen, ${ }^{3} \mathrm{O}$ Erdeve, ${ }^{1 B}$ Sarıdas, ${ }^{2} \mathrm{E}$ Canpolat, ${ }^{4} \mathrm{U}$ Dilmen. ${ }^{1}$ Developmental Behavioral Pediatrics Unit; ${ }^{2}$ Neonatology, Zekai Tahir Burak Maternity and Teaching Hospital; ${ }^{3}$ Neonatology, Ankara University, Faculty of Medicine; ${ }^{4}$ Neonatology, Zekai Tahir Burak Maternity and Teaching Hospital, YIIdırım Beyazit University Department of Pediatrics, Ankara, Turkey

Objective Although neurodevelopmental outcomes for management of patent ductus arteriosus with intravenous indomethacin and ibuprofen are known, there has been lack of data on long term effects of oral ibuprofen in the literature.

Method To assess the neurodevelopmental outcomes at 18-24 months' corrected age, we conducted a prospective follow-up study of 99 infants with birth weight $\leq 1500 \mathrm{~g}$ and gestational age $\leq 32$ weeks who recieved either oral or intravenous ibuprofen for patent ductus arteriosus.1) Moderate/severe cerebral palsy with functional deficits, 2) bilateral hearing loss and blindness in either eye, and 3) mental developmental index score or psychomotor index score less than 70 were defined as abnormal neurologic, neurosensory and cognitive outcomes, respectively.

Results At 18-24 months' corrected age, neurodevelopmental outcomes of $30(60.0 \%)$ among 50 subjects who received oral ibuprofen were compared with 27 (55.1\%) of 49 who received intravenous ibuprofen by certified and experienced examiners who were blinded to the groups. The results revealed that long term outcomes did not significantly differ among treatment regimes.

Conclusion Preterm infants who were treated with oral ibuprofen for patent ductus arteriosus have similar neurological, neurosensory and cognitive outcomes with patients who recieved intravenous ibuprofen at 18-24 months' corrected age.

\section{LONGITUDINAL FOLLOW UP IN A COHORT OF ELBW/ VLBW INFANTS IN THE CONTEXT OF THE BAYLEY-III STANDARDIZATION IN GREECE}

doi:10.1136/archdischild-2012-302724.1245

'V Soubasi, ${ }^{2} \mathrm{~K}$ Velikos, ${ }^{2} \mathrm{M}$ Michalettou, ${ }^{2} \mathrm{~V}$ Papadopoulou, ${ }^{3} \mathrm{D}$ Zafeiriou, ${ }^{1} \mathrm{P}$ Papakyriakidou 'V Drossou. 11st Neonatal Clinic, Ippokration General Hospital, Aristotle University of Thessaloniki; ${ }^{2}$ Dept of Child Psychiatry, Ippokration General Hospital; ${ }^{3} 1$ st Dept of Paediatrics, Ippokration General Hospital, Aristotle University of Thessaloniki, Thessaloníki, Greece

Background It is not clear whether the Bayley III is overestimat ing cognitive performance or it is a more valid assessment of emerging cognitive skills than the older edition II(Vohr B 2012).

Aim To compare the developmental profile in a cohort of premature infants in two different time points in order to assess the stability of the results, in the context of the Bayley-III standardization in Greece.

Methods Bayley-III scales(cognitive, language, motor, socialemotional, and adaptive behavior), were administered in 103 preterm infants ( $\leq 32$ weeks) at a mean age of $12 \mathrm{mo}$ and $24 \mathrm{mo}$. Infants were divided in 2 groups based to their GA: $A(n=48,24-28 w k s)$, 